\title{
Bilateral lingual-facial trunk: anatomic and clinical implications
}

\author{
T. Troupis ${ }^{1}$, A. Michalinos ${ }^{1}$, J. Kakisis², K. Natsis ${ }^{3}$, G. Sofidis ${ }^{3}$, P. Skandalakis ${ }^{1}$ \\ ${ }^{1}$ Department of Anatomy, Faculty of Medicine, National and Kapodistrian University of Athens, Greece \\ 2Department of Vascular Surgery, "Attikon" University Hospital, National and Kapodistrian University of Athens, Greece \\ ${ }^{3}$ Department of Anatomy, Faculty of Medicine, Aristotle University of Thessaloniki, Greece
}

[Received 4 November 2014; Accepted 12 December 2014]

\begin{abstract}
Common origin of lingual and facial artery is a relatively frequent anatomic variation. Instead, bilateral lingual-facial trunk has been described only sparsely in the literature. In this report authors describe and analyse a case of bilateral common lingual-facial trunk in the context of its anatomical, clinical and embryological implications. We also describe possible consequences in performance of elective and emergent surgical operations and modification in surgical techniques that should be considered. We believe that surgeons should be suspicious for this variation's existence and keep alternative solutions in their armentarium. (Folia Morphol 2015; 74, 4: 548-551)
\end{abstract}

Key words: lingual-facial trunk, post-tonsillectomy haemorrhage, endovascular occlusion

\section{INTRODUCTION}

Arterial vascularisation of head and neck region originates from common carotid artery (CCA), internal carotid artery (ICA) and external carotid artery (ECA). Front branches of the ECA are the superior thyroid artery (STA), lingual artery (LA), and facial artery (FA) that vascularise the thyroid gland, tongue, and face, respectively [3].

Anatomic variations in origin, course and distribution of those branches are common. While most of them are clinically insignificant, they might affect certain diseases' clinical image or alter surgical technique in case of both elective operations (tumour removal operation, radical neck dissection, flaps used in plastic surgery) and surgical emergencies $[1,6]$.

This report describes a rare case of bilateral lingual-facial trunk (LFT) discovered during dissection of a male Caucasian cadaver. We describe analytically the characteristics of this rare variation and attempt to investigate its anatomical, clinical and embryological implications.

\section{CASE REPORT}

During dissection of a Caucasian male cadaver at anatomy course at laboratory of anatomy, Faculty of Medicine, National and Kapodistrian University of Athens, Greece, anatomic variations of carotid artery branches were encountered. Primary dissection was made by students, for educative purposes, while further dissection for research reasons by authors.

After removing the skin and the subcutaneous tissue, we proceeded to the dissection of the musculature of the neck region. We defined the borders of the carotid triangle and in it we dissected the CCA, ECA, ICA, and the anterior branches of the ECA at the area, i.e., STA, LA, and FA.

Particularities found (bilateral LFT) were carefully dissected, photographed and measured with the use of a calliper. During measurement, no tension was exercised on arteries and their courses were kept as dissected. Sizes measured were the LFT's distance from the carotid bifurcation and STA as was trunk's length and diameter. The same measurements were 


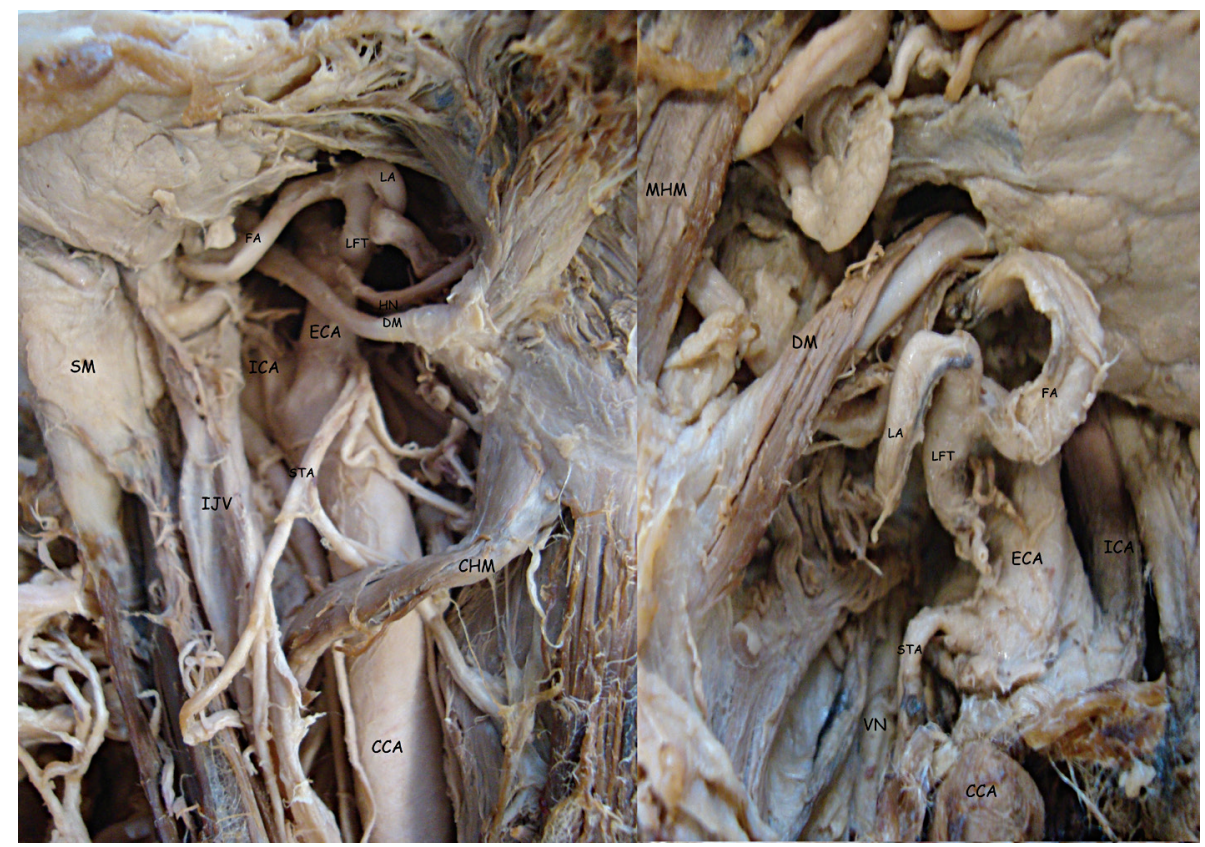

Figure 1. Bilateral lingual-facial trunk, right and left side; CCA — common carotid artery; ICA — internal carotid artery; ECA — external carotid artery; STA — superior thyroid artery; LA — lingual artery; FA — facial artery; LFT — lingual-facial trunk; IJV — internal jugular vein; $\mathrm{HN}$ - hypoglossal nerve; VN — vagus nerve; SM — sternocleidomastoid muscle; DM — digastric muscle; $\mathrm{CHM}$ — coracohyoid muscle; $\mathrm{MHM}$ - mylohyoid muscle.

performed on the other side. For any measurement, cadavers remained in the supine position, as described in textbooks.

Lingual artery and FA bilaterally arose combined in a common trunk (Fig. 1). On the right side, LFT's diameter was measured at $3.1 \mathrm{~mm}$, its origin from carotid bifurcation at $7.6 \mathrm{~mm}$ and its length before dividing into LA and FA at $3.1 \mathrm{~mm}$. On the left side, LFT's diameter was measured at $3.3 \mathrm{~mm}$, its origin from carotid bifurcation (CB) at $6.8 \mathrm{~mm}$ and its length at $3.4 \mathrm{~mm}$. No other variations concerning cervical region were encountered.

\section{DISCUSSION}

Lingual-facial trunk is a relative rare variation, its incidence ranging between $7 \%$ and $20 \%[2,5]$. Its morphometric characteristics have been only rarely described, usually in the context of a larger study. Its average length before division at LA and FA is measured at $9.4 \pm 1.7 \mathrm{~mm}$ at the right side and $7.6 \pm 1.3 \mathrm{~mm}$ on the left side, ranging $4.3-11.4 \mathrm{~mm}$ $[5,10,13]$. Its distance from $C B$ ranges $5-40 \mathrm{~mm}[7,13]$ and its diameter approximately $2 \mathrm{~mm}$ [5]. The latter is surprising because LFT's diameter does not significantly differ from diameter of LA and FA $(1.5 \mathrm{~mm}$ and $2 \mathrm{~mm}$, respectively [3]), while it should since it has to provide their combined flow. Its embryologic origin is obsolete. It can be considered as a variation of the origin of the external carotid branches, a variation on the facial artery origin, or a variation of LA origin. Another hypothesis is that such trunks can be created by remnants of the second aortic arch in the foetus. None of this can determine its embryological origin, but still its existence can be proved critical in certain operations such as stemming of uncontrolled bleeding, intraoral biopsy, tumour invasion, and maxillofacial trauma [6]. Bilateral LFTs has been described only by Fazan et al. [5] in their study including 42 cases. Only 2 cases were found and calculated incidence was $4.8 \%$. To the best of our knowledge, no other cases of bilateral LFT have been described.

Anatomic variations are of critical importance in every operation but adequate time for careful dissection and preoperative diagnosis diminish the possibility of an error. Angiography is currently the "gold standard" for preoperative visualisation of cervical area vasculature yet other, less invasive modalities are of interest. Cappabianca et al. [2] in a radiologic study successfully characterised cervical area arterial anatomic variations with the use of computed tomography angiography and magnetic resonance angiography. Except from describing some relative 
common anatomic variations, authors also succeeded in describing analytically rarer ones.

This is not the case in surgical emergencies, like uncontrolled intraoral bleeding since there is often inadequate time for radiological means. Extraoral ligation of LA is an appropriate and effective yet dangerous procedure for this situation. It might occur after maxillofacial trauma, intraoral tumour biopsy, automatically after tumour invasion or after surgical debulking and even after minor procedures including tonsillectomy. First line of treatment consists of local measures like direct pressure, coagulation and vessels ligation at surgery field [12]. However, intraoral local situation, like bleeding itself, oedema or previous attempts, compromise success of local means. If these measures fail, extraoral ligation or endovascular occlusion of LA is necessary for haemorrhage control. Extraoral ligation, while very effective, has a number of potential complications including injury of the superior laryngeal or vagus nerve, cerebrovascular accidents and diminished vascular reserve in the arterial distribution of the ligated vessels. Cerebrovascular accidents are also cardinal complications of endovascular occlusion. For both modalities, existence of LFT can lead to treatment failure due to difficulties in identifying vasculature, especially in an emergency basis or add morbidity (since occlusion of LFT will also cause occlusion of FA). Consequences from ligation of FA should be minimum due to rich anastomotic vasculature from branches of ipsilateral external carotid artery or contra lateral FA. In a number or patients with compromised vasculature due to local (e.g. trauma) or systemic diseases, ligation of LFT could be hazardous.

Post-tonsillectomy haemorrhage consists $10 \%$ of post-surgical haemorrhages in otorhinolaryngology [9, 12]. Rich vasculature of tonsils derives from ascending palatine artery, tonsillar artery (branches of FA) and dorsal lingual artery (branch of LA). While extraoral ligation of LA or even ECA is useful for uncontrolled post-tonsillectomy haemorrhage, intravascular occlusion is proposed as first-line treatment. Occlusion of LFT for post-tonsillectomy haemorrhage has already been described in the literature [1].

Bilateral neck dissection is currently an operation reserved for patients with advanced cancer of cervical and facial area due to its high morbidity and mortality. Usual complications are postoperative bleeding, oedema and chylothorax due to thoracic duct injury $[4,8]$. While preservation of vascular elements should always be attempted, arteries excision is necessary in case of infiltration of neoplastic tissue, a procedure called extended radical neck dissection. Ischaemic complications are described after extended radical neck dissection, usually cerebrovascular accidents and defects of visual function $[11,14]$. Selective ischaemia of the tongue or the face is a theoretical possibility due to the rich anastomotic vasculature between lingual and FA and, to the best of our knowledge, it has never been described. Still this is a possibility that cannot be neglected in the case of a bilateral LFT. In doubtful cases surgeon should be aware and weight the possibility of possible ischaemia.

Selective or radial dissection of the neck region is necessary for an extremely large number of procedures of general surgery, maxillofacial surgery, vascular surgery, otolaryngology and plastic surgery such as thyroidectomy, parathyroidectomy, extraction of thyroglossal or brachial cysts, carotid endarterectomy, aneurysms management, tracheotomy, oesophageal procedures, tumour extraction, cleaning of metastatic lymph nodes, or trauma management. During all these procedures, most feared complication is rupture of ECA or one of its branches or extensive tissue ischaemia due to inappropriate variation. Existence of an LFT and even more a bilateral LFT bears both these complication. Detailed knowledge of anatomic variations and implications they cause in surgical technique, careful preoperative staging with all appropriate means and meticulous dissection during procedures are most important weapons in a surgeon's armentarium.

\section{REFERENCES}

1. Atmaca S, Belet U, Baris S (2014) Post-tonsillectomy pseudoaneurysm of the linguofacial trunk: an ENT surgeon's nightmare. Int J Pediatr Otorhinolaryngol Extra, 7: 12-14.

2. Cappabianca $S$, Scuotto $A$, laselli F, Pignatelli $N$, Urraro $F$, Sarti G, Montemarano N, Graci R, Rotondo A (2012) Computed tomography and magnetic resonance angiography in the evaluation of aberrant origin of the external carotid artery branches. SARA, 34: 393-399.

3. Drake RL, Mitchell AWM, Vogl W (2005) Gray's anatomy for students. Elsevier Churchill Livingstone, Philadelphia.

4. Dua A, Desai SS (2014) Utility of internal jugular vein reconstruction in modified radical neck dissection. Vascular, 22: 81-84.

5. Fazan VPS, Silva JHN, Borges CT, Ribeiro RA, Caetano AG, Filho OAR (2008) An anatomical study on the lingual-facial trunk. SARA, 31: 267-270.

6. Homze EJ, Harn SD, Bavitz BJ (1997) Extraoral ligation of the lingual artery: an anatomic study. Oral Surg Oral Med Oral Pathol Oral Radiol Endod, 83: 321-324.

7. Lucev N, Bobinac D, Maric I, Drescik I (2000) Variations of the great arteries in the carotid triangle. Otolaryngol Head Neck Surg, 122: 590-591. 
8. Masud MK, Ahmad SM, Karim MA Ferdouse F, Fakir A, Hanif M, Abdulah M, Roy A (2014) Complications of neck dissection at a tertiary level hospital: study of 30 cases. Mymensingh Med J, 23: 658-666.

9. Opatowsky MJ, Browne JD, McGuirt Jr WF, Morris PP (2001) Endovascular treatment of hemorrhage after tonsillectomy in children. AJNR, 22: 713-716.

10. Ozgur Z, Govsa F, Ozgur T (2008) Assessment of origin characteristics of the front branches of the external carotid artery. J Craniofac Surg, 19: 1159-1166.

11. Robbins KT, Clayman G, Levine PA, Meddina J, Sessior R, Shaha A, Som P, Wolf G (2002) Neck dissection classification update: revisions proposed by the American Head and Neck Society and the American Academy of
Otolaryngology-Head and Neck Surgery. Arch Otolaryngol Head Neck Surg, 128: 751-758.

12. Schrock A, Jakob M, Strach K, Pump B, Gerstner A, Wilheim K, Urbach H, Bootz F, Grechus S (2012) Transarterial endovascular treatment in the management of life-threatening intraand postoperative haemorrhages after otorhinolaryngological surgery. Eur Arch Oto-Rhino-Laryngol, 269: 1677-1683.

13. Troupis TG, Dimitroulis D, Paraschos A, Michalinos A, Protogerou V, Vlasis K, Troupis G, Skandalakis P (2011) Lingual and facial arteries arising from the external carotid artery in a common trunk. Am Surg, 77: 151-154.

14. Worrell L, Rowe M, Petti G (2002) Amaurosis: a complication of bilateral radical neck dissection. Am J Otolaryngol, 23: $56-59$. 